\title{
Review: thioridazine is not more effective than placebo or other neuroleptic drugs in elderly patients with dementia
}

\author{
Kirchner V,Kelly CA, Harvey RJ. A systematic review of the evidence for the safety and efficacy of thioridazine in dementia. \\ (Cochrane Review, latest version 14 Aug, 1998). In: Cochrane Library. Oxford: Update Software.
}

\section{Question}

In elderly patients with dementia, is thioridazine effective and safe and does it improve cognitive outcomes?

\section{Data sources}

Studies were identified by searching Medline, EMBASE/ Excerpta Medica, PsycLIT, CINAHL, and the Cochrane Group Register of Clinical trials using the terms thioridazine, Melleril, dementia, and old age; and by reviewing additional published and unpublished studies.

\section{Study selection}

Studies were selected if they were randomised trials of thioridazine (used for $\geqslant 1$ dose) compared with placebo, no treatment, an alternative drug, or behavioural intervention in patients with a degenerative dementia. Studies were excluded from the analysis if treatment allocation was not concealed.

\section{Data extraction}

Data were extracted on patient characteristics, allocation procedure, study quality, blinding, interventions used, dropouts, analysis methods, drug safety, institutionalisation, death, and pretreatment and post-treatment dementia assessment scores. Dementia was assessed using behavioural, clinical global impression, functional performance, and cognitive scales.

\section{Main results}

51 studies were identified. 10 studies met the inclusion criteria; 7 studies had sufficient data for inclusion in the analysis. These 7 studies (published between 1973 and 1992) were double blind; ranged from 3 to 8 weeks duration, included between 30 and 610 patients with mean ages between 73 and 80 years; used thioridazine doses between 10 and $200 \mathrm{mg}$; and included comparisons of thioridazine with placebo, diazepam, loxapine, zuclopenthixol, etoperidone, and chlormethiazole. In 1 study, more patients who received thioridazine than those who received placebo had improved anxiety scores based on an assessment of anxious mood, tension, fear, and insomnia $\{79 \%$ v $41 \%$, $\mathrm{p}<0.001\}^{*}$ and agitation, intellect, depressed mood, and behaviour at interview $\{74 \% \text { v } 42 \%, \mathrm{p}<0.001\}^{*}$; no difference existed for clinical global change or adverse effects. In 1 study, more patients who received thioridazine than those who received diazepam had improved anxiety scores based on an assessment of agitation, intellect, depressed mood, and behaviour at interview $\{77 \% v 65 \%, \mathrm{p}=0.03\}$; no difference existed for clinical global change or adverse effects. In 1 study, fewer patients who received thioridazine than those who received chlormethiazole had improved behavioural rating scores for items of confusion and alertness $\{20 \% v 47 \%, \mathrm{p}=0.03\}^{*}$, continence $\{10 \%$ v $37 \%$, $\mathrm{p}=0.01\}^{*}$, and nocturnal awakening $\{47 \% \approx 80 \%, \mathrm{p}=0.007\}^{*}$; and patients who received thioridazine had increased dizziness $\{23 \%$ v $3 \%, \mathrm{p}=0.02\} *$. Thioridazine was not more effective than etoperidone, loxapine, or zuclopenthixol.

\section{Conclusions}

Thioridazine is not more effective than placebo or other neuroleptic drugs for improving the behavioural, global clinical, or cognitive states of elderly patients with dementia. Thioridazine reduced anxiety only when compared with placebo or diazepam and led to worsened behavioural scores and increased dizziness when compared with chlormethiazole.

* p value calculated from data in article.

Sources of funding: no external funding.

For correspondence:Dr Richard Harvey, Dementia Research Group, Institute of Neurology and Division of Neurosciences, Imperial College, The National Hospital for Neurology and Neurosurgery, Oueen Square, London WC1N 3BG, UK. Fax +44 (0)171 2090182

\section{Commentary}

The treatment of behavioural abnormalities in dementia is a perennial quandary for neuropsychiatrists. Treatment strategies for these problems are largely governed by testimonial data because few controlled trials exist to guide the way. The situation is quite understandable; controlled studies are difficult to do in light of diagnostic heterogeneity, concomitant medical illnesses, and informed consent issues. Furthermore, there are probably many different neurobiological reasons why patients suffering from a given type of dementia would develop agitation, aggression, and disruptive behaviour.

Although there is widespread use of thioridazine for agitation in dementia, the meta-analysis of Kirchner et al would suggest cautious use of this compound in the future. The use of this meta-analysis as a basis for conclusions, however, is problematic because it draws from studies with a diverse mixture of treatment comparisons, diagnostic groups, and rating scales. Surprisingly, thioridazine is superior to placebo or diazepam only on some items from the Hamilton Anxiety Scale, but not on global measures of clinical improvement. This may indicate that ratings obtained based on patients' internal experience and behaviour with an examiner improve more than global measures of their behaviour in groups. Obviously, one cannot conclude that thioridazine is a superior anxiolytic compared with diazepam unless one controls for the level of sedation induced by the 2 drugs. It appears that several non-sedating neuroleptics are as effective in reducing agitation as thioridazine, which would suggest that the sedat- ing properties of thioridazine and related drugs are not as important as once believed in reducing anxiety and agitation. The important question inevitably becomes whether the risk of extrapyramidal side effects (EPSs) with non-sedating neuroleptics is greater than the risk of cardiovascular and other side effects with drugs such as thioridazine. This question cannot be answered reliably from study data and must still be decided on a case by case basis by informed clinicians.

The study of Devanand $e t$ al is more helpful. In addition to beginning with a decent sample size $(\mathrm{n}=71)$, the authors excluded dementias other than probable Alzheimer's disease; used clearly operationalised entry criteria for the presence of psychosis, aggression, and agitation; (commentary continued on page 48 ) 


\title{
Haloperidol, 2-3 mg/day, decreased psychosis and disruptive behaviours in Alzheimer's disease
}

\author{
Devanand DP, Marder K, Michaels KS, et al. A randomized, placebo-controlled dose-comparison trial of haloperidol for psychosis \\ and disruptive behaviors in Alzheimer's disease. Am J Psychiatry 1998 Nov;155:1512-20.
}

\section{Question}

In patients with Alzheimer's disease $(\mathrm{AD})$ and psychosis or disruptive behaviour, how effective and safe are 2 doses of haloperidol compared with placebo?

\section{Design}

6 week randomised, double blind, placebo controlled, dose comparison trial (phase A) followed by a subsequent 6 week crossover phase (phase B).

\section{Setting}

Memory disorders clinic in New York State, USA.

\section{Patients}

71 outpatients (mean age $72 \mathrm{y}, 65 \%$ women) with DSM-III-R criteria for dementia and the criteria for probable $\mathrm{AD}$ of the National Institute of Neurological and Communicative Disorders and Stroke and the Alzheimer's Disease and Related Disorders Association, and either psychosis or disruptive behaviours. Exclusion criteria were drug or alcohol dependence; stroke; or a history of other causes of dementia including head trauma, Parkinson's disease, Huntington's disease and multiple sclerosis. 60 patients $(85 \%)$ completed phase A and $69 \%$ completed phase B.

\section{Intervention}

All patients received placebo for the initial 1 week single blind phase. Patients were then randomised to 6 weeks of standard dose haloperidol (2-3 mg/d), low dose haloperidol (0.50-0.75 $\mathrm{mg} / \mathrm{d}$ ), or placebo (phase A). After 6 weeks, patients in the 2 haloperidol groups received placebo and patients who received placebo were randomly assigned to the 2 doses of haloperidol (phase B).

\section{Main outcome measures}

The primary outcome measures for effectiveness were the Brief Psychiatric Rating Scale (BPRS) psychosis and hostile suspiciousness factor scores, the Behavioral Syndromes Scale for Dementia, item scores for psychomotor agitation and physical aggression, and the sum of 3 target symptoms scores identified from the psychosis and disorganisation items of the Schedule for Affective Disorders and Schizophrenia (SADS) scale at study entry. Side effects were also assessed.

\begin{abstract}
Main results
During phase A, standard dose haloperidol was superior to low dose haloperidol and placebo for BPRS psychosis factor scores $(p<0.05)$ and psychomotor agitation $(p<0.03)$. No difference existed between the haloperidol groups on target symptoms of the SADS. Extrapyramidal side effects (EPSs) were slightly greater with standard dose haloperidol than low dose $(\mathrm{p}<0.08)$; $20 \%$ of patients in the standard dose group developed moderate to severe side effects. Low dose haloperidol did not differ from placebo for effectiveness or side effects.
\end{abstract}

\section{Conclusions}

Haloperidol $(2-3 \mathrm{mg} / \mathrm{d})$ decreased psychosis and disruptive behaviours in Alzheimer's disease although 20\% of patients developed moderate to severe extrapyramidal side effects.

Sources of funding: National Institute of Mental Health; National Institute on Aging; the Banbury Fund.

For correspondence: Dr D P Devanand, Biological Psychiatry, Box 126, New York State Psychiatric Institute, 722 West 168th Street, New York, NY 10032, USA. Fax +1 2125435854. (commentary continued from page 47) and looked at the data with completer and intent to treat analyses. They found that $2-3 \mathrm{mg} /$ day of haloperidol is superior to $0.50-0.75 \mathrm{mg} /$ day haloperidol and to placebo. It is now known that $2 \mathrm{mg}$ of haloperidol produces $50 \%$ to $70 \%$ saturation of striatal $\mathrm{D}_{2}$ receptors, a level of saturation associated with good clinical response in schizophrenia and below the threshold of $70 \%$ to $80 \% \mathrm{D}_{2}$ occupancy for the emergence of EPS. ${ }^{1}$ This study, in combination with previous reports on schizophrenia, would suggest that $\mathrm{D}_{2}$ antagonism does indeed have relevance for the treatment of psychosis, regardless of the aetiology.

On the other hand, EPSs are a significant limitation of haloperidol in this study. In patients with Alzheimer's disease, $2-3 \mathrm{mg}$ /day of haloperidol may produce a greater degree of $\mathrm{D}_{2}$ occupancy, if there has been degenerative change in the nigrostriatal dopamine pathway, and consequently more EPSs. More importantly, improvement in outcome measurements for psychosis, agitation, and aggression in future studies should be controlled for the severity of EPSs. In the study by Devanand et al, however, clinical improvement was not associated with increased EPSs. It is not hard to imagine that psychosis, aggression, and agitation can appear improved simply because patients have become parkinsonian. Controlling the data in this way will have important implications for understanding the pathophysiology of psychosis and for evaluat- ing more accurately the effects of so called "atypical" neuroleptics.

Finally, the finding that blood concentrations of haloperidol are better correlated with clinical outcome than oral dose concentration is an interesting aspect of this study. The authors' suggestion of a 1 $\mathrm{mg} /$ day starting dose with monitoring of blood concentrations would appear to be a sound recommendation based on the available evidence.

Michael Knable, DO Stanley Foundation Bethesda, Maryland, USA

1 Kapur S, Remington G, Jones C, et al. High levels of dopamine D2 receptor occupancy with low-dose halperidol treatment: a PET study. Am J Psychiatry 1996;153:948-50. 